\title{
Data from emergency medicine palliative care access (EMPallA): a randomized controlled trial comparing the effectiveness of specialty outpatient versus telephonic palliative care of older adults with advanced illness presenting to the emergency department
}

Abigail M. Schmucker ${ }^{1}$, Mara Flannery ${ }^{2 *}$, Jeanne Cho ${ }^{2}$, Keith S. Goldfeld ${ }^{3}$, Corita Grudzen ${ }^{2,3}$ and The EMPallA Investigators

\begin{abstract}
Background: The Emergency Medicine Palliative Care Access (EMPallA) trial is a large, multicenter, parallel, two-arm randomized controlled trial in emergency department (ED) patients comparing two models of palliative care: nurseled telephonic case management and specialty, outpatient palliative care. This report aims to: 1) report baseline demographic and quality of life (QOL) data for the EMPallA cohort, 2) identify the association between illness type and baseline QOL while controlling for other factors, and 3) explore baseline relationships between illness type, symptom burden, and loneliness.

Methods: Patients aged 50+ years with advanced cancer (metastatic solid tumor) or end-stage organ failure (New York Heart Association Class III or IV heart failure, end stage renal disease with glomerular filtration rate $<15 \mathrm{~mL} / \mathrm{min} / \mathrm{m}^{2}$, or Global Initiative for Chronic Obstructive Lung Disease Stage III, IV, or oxygen-dependent chronic obstructive pulmonary disease defined as $\mathrm{FEV}_{1}<50 \%$ ) are eligible for enrollment. Baseline data includes self-reported demographics, QOL measured by the Functional Assessment of Cancer Therapy-General (FACT-G), loneliness measured by the Three-ltem UCLA Loneliness Scale, and symptom burden measured by the Edmonton Revised Symptom Assessment Scale. Descriptive statistics were used to analyze demographic variables, a linear regression model measured the importance of illness type in predicting QOL, and chi-square tests of independence were used to quantify relationships between illness type, symptom burden, and loneliness.
\end{abstract}

\footnotetext{
*Correspondence: Mara.Flannery@nyulangone.org

${ }^{2}$ Ronald O. Perelman Department of Emergency Medicine, NYU School of Medicine, New York University Langone Health, 227 E 30th Street, First Floor, New York, NY 10016, USA

Full list of author information is available at the end of the article
}

(c) The Author(s). 2021 Open Access This article is licensed under a Creative Commons Attribution 4.0 International License, which permits use, sharing, adaptation, distribution and reproduction in any medium or format, as long as you give appropriate credit to the original author(s) and the source, provide a link to the Creative Commons licence, and indicate if changes were made. The images or other third party material in this article are included in the article's Creative Commons licence, unless indicated otherwise in a credit line to the material. If material is not included in the article's Creative Commons licence and your intended use is not permitted by statutory regulation or exceeds the permitted use, you will need to obtain permission directly from the copyright holder. To view a copy of this licence, visit http://creativecommons.org/licenses/by/4.0/ The Creative Commons Public Domain Dedication waiver (http://creativecommons.org/publicdomain/zero/1.0/) applies to the data made available in this article, unless otherwise stated in a credit line to the data. 
Results: Between April 2018 and April 3, 2020, 500 patients were enrolled. On average, end-stage organ failure patients had lower QOL as measured by the FACT-G scale than cancer patients with an estimated difference of 9.6 points (95\% Cl: 5.9, 13.3), and patients with multiple conditions had a further reduction of 7.4 points ( $95 \%$ Cl: $2.4,12.5)$, when adjusting for age, education level, race, sex, immigrant status, presence of a caregiver, and hospital setting. Symptom burden and loneliness were greater in end-stage organ failure than in cancer.

Conclusions: The EMPallA trial is enrolling a diverse sample of ED patients. Differences by illness type in QOL, symptom burden, and loneliness demonstrate how distinct disease trajectories manifest in the ED.

Trial registration: Clinicaltrials.gov identifier: NCT03325985. Registered October 30, 2017.

Keywords: Palliative care, Randomized controlled trial, Geriatrics, Advanced cancer, End-stage organ failure, Functional decline, Patient-reported outcomes, Quality of life

\section{Background}

Emergency departments are frequently visited by severely ill patients approaching the end of life; in a study of older adults who died between 1992 and 2006, 75\% visited the emergency department during the last six months of life [1]. Although the focus of emergency medicine has traditionally been the diagnosis and treatment of acute illnesses and injuries, emergency clinicians have identified a growing need for palliative care interventions in the emergency setting to address the symptoms and stresses of advanced chronic illnesses [2, 3].

Palliative care is defined by the World Health Organization as "an approach that improves the quality of life (QOL) of patients and their families facing the problems associated with life-threatening illness, through the prevention and relief of suffering by means of early identification and impeccable assessment and treatment of pain and other problems, physical, psychosocial, and spiritual." [4] Palliative care has been shown to improve patients' symptoms and QOL across a broad range of serious, life-limiting illnesses. Patients receiving palliative care are often able to remain cared for and supported at home, resulting in greater patient and family satisfaction and less prolonged grief and post-traumatic stress disorder among bereaved family members [5-10]. Palliative care also decreases healthcare-related costs by reducing unnecessary hospitalizations, interventions, and avoidable ED and intensive care [11-15].

In cancer patients, randomized controlled trials of palliative care interventions have shown better QOL and mood, as well as improved symptom management and patient satisfaction, with palliative care in addition to standard care $[12,16]$. Palliative care has its roots in the care of cancer patients at the end of life, but increasing studies support its potential to benefit patients with endstage organ failure, such as chronic obstructive pulmonary disease (COPD), congestive heart failure (CHF) and end-stage renal disease (ESRD), which can also cause physical, psychosocial, and spiritual distress $[17,18]$.

While both cancer and end-stage organ failure patients stand to benefit from palliative care, the illness trajectories associated with advanced cancer differ from those of organ failure [19]. Cancer patients commonly have a short period of evident decline before death; however, patients with endstage organ failure have a pattern of long-term decline with episodes of worsening and remission [18]. Efforts to integrate palliative care screening and assessment into the emergency department have yielded positive outcomes, yet further research is needed to identify specific effective interventions [20]. Understanding aspects of patients' experiences of advanced chronic diseases, such as quality of life and symptom burden, at the time of their presentation to the emergency department could assist emergency providers as they develop patient-centered treatment plans. We are not aware of any literature describing how the distinct trajectories of advanced cancer and end-stage organ failure patients manifest in the ED.

This paper reports on a large, multicenter, parallel, two-arm randomized controlled trial in ED patients comparing two established models of palliative care: nurse-led telephonic case management and specialty, outpatient palliative care. The objectives of this paper are to: 1) report preliminary baseline demographic and QOL data for the EMPallA trial cohort, 2) identify the association between illness type and baseline QOL while controlling for other baseline factors, and 3) explore the baseline relationships between illness type, symptom burden, and loneliness.

\section{Methods}

\section{Study design}

The Emergency Medicine Palliative Care Access (EMPallA) trial was approved by the Institutional Review Boards (IRBs) across all study sites. This study is funded through the Patient-Centered Outcomes Research Institute (PCORI). We registered this randomized controlled trial (RCT) in the international trial register (ClinicalTrials.gov: Identifier NCT03325985). This study adheres to Consolidated Standards of Reporting Trials (CONSORT) guidelines. See Supplement 1 for CONSORT checklist. For further details please refer to our protocol paper [21]. 


\section{Study setting and population}

This RCT began recruitment in April 2018 and is currently enrolling at 18 emergency department (ED) sites across the United States (US), with locations representing the geographic diversity of the country. Patients considered for enrollment in this study comprise adults aged 50 years or older, who have advanced cancer (metastatic solid tumor) or end-stage organ failure (New York Heart Association class III or IV heart failure, end stage renal disease with glomerular filtration rate $<15 \mathrm{~mL} /$ $\mathrm{min} / \mathrm{m}^{2}$ or on dialysis, or global initiative for chronic obstructive lung disease stage III, IV or oxygendependent), reside within the geographical area, have a working telephone, and have health insurance. Exclusion criteria include not speaking English or Spanish, having dementia documented in the electronic health record (EHR) problem list, having received hospice services or two or more palliative care visits in the last six months, residing in a long-term care facility, or being admitted to the hospital for more than $48 \mathrm{~h}$ post ED encounter.

\section{Study protocol}

Enrollment of patients is ongoing as our target sample size is 1350 patients, but for this cohort occurred between April 2018 and April 3, 2020. Screening and enrollment take place seven days per week, $24 \mathrm{~h}$ per day. Research assistants (RAs) check the ED and observation unit electronic track boards to identify patients with qualifying medical conditions, and then review the patients' EHR to confirm inclusion criteria are met. RAs then approach patients and conduct face-to-face interviews to confirm all eligibility criteria are met. RAs complete written informed consent and Health Insurance Portability and Accountability Act authorization and conduct a survey to gather baseline data. For Spanish-speakers, a language-appropriate consent form is used and either bilingual, certified study staff or noninvestigator, hospital-employed, trained interpreters assist in acquisition of informed consent. Once enrolled, the coordinating site performs two-arm randomization (outpatient specialty palliative care vs telephonic nursedelivered palliative care) stratified by site and illness type (cancer vs. end-stage organ failure).

The central team ensures uniform application of the methods across sites via an initial site visit, detailed Standard Operating Procedures (SOPs), standardized training of all new staff, as well as consistent re-training, and practice of the study pitch via tele-conference with central team members. Data collection, management and randomization occur using in a central REDCap database hosted at NYU School of Medicine to ensure consistency $[22,23]$. Additional details are highlighted in the study protocol paper [21].

\section{Measures}

Research assistants collect primary and secondary outcome data as well as demographic variables via face-toface bedside interview or EHR at baseline. Demographic data include sex, race, ethnicity, income, education, religion, marital status, insurance, birth country, residency type, and language.

The primary study outcome is change in patient QOL from enrollment to six months, as measured by the Functional Assessment of Cancer Therapy-General (FACT-G, Version 4). QOL is a well-established outcome measure in palliative care research, $[24,25]$ and the FACT-G has been validated and used extensively to assess chronic disease therapy in many serious illnesses [24]. It has 28 items in a five-point Likert scoring scale from "0" (Not at all) to "4" (Very much) which assess QOL across four domains: physical, social/family, emotional, and functional. We followed the standard FACTG Version 4 scoring algorithm to obtain both subscale and total FACT-G scores. We broke down FACT-G scores by domain to obtain a subscale score (possible range $0-28$ for physical, social/family, and functional; 0 24 for emotional), and summed the subscale scores to obtain an overall QOL score (possible range 0-108). Higher FACT-G scores in each domain as well as overall indicate better QOL.

Secondary outcomes in patients include loneliness, as measured by change in Three-Item UCLA Loneliness Scale from enrollment to 6 months [26, 27]; and symptom burden, as measured by change in Edmonton Revised Symptom Assessment Scale (ESAS-r) from enrollment to 6 months [28]. The Three-Item Loneliness Scale comprises a three-by-three Likert scale, with higher score indicating more loneliness. To maximize validity of a three-item scale, we assessed each question (lacking companionship, feeling left out, feeling isolated from others) separately and did not sum the responses for a total loneliness score.

The ESAS-r comprises nine common symptoms rated in severity on an 11-point scale plus space to rate additional symptoms; higher scores indicate greater symptom burden. Studies of the older version of the same scale (ESAS) have shown that a more general picture of symptom severity can be gained by summing individual symptom scores for a total symptom burden score, reported on a scale from 0 to 100 . Total symptom burden can therefore be assessed as absent (0), mild [1-30], moderate (31-60), or severe (61-100), and clinical significance is previously defined as a total symptom score of 31 or greater [29, 30]. For the purposes of this baseline analysis, we summed items to obtain a total symptom score but did not look at individual symptom responses (e.g. pain). 


\section{Data analysis}

We described baseline socio-demographic characteristics and functional status for patients enrolled in the study through April 2020. Means and standard deviations were calculated for continuous variables, and frequencies and percentages were calculated for categorical variables.

To assess the strength of the relationship of disease condition and QOL as measured by the FACT-G, we first used data visualization. We estimated a linear regression model to examine the importance of illness group membership in predicting overall FACT-G score, adjusting for age, education level, race, sex, immigrant status, the presence of a caregiver, comorbid illness, and hospital setting. All covariates were pre-determined a priori and included in the model based on our belief that they might be potential predictors of FACT-G scores.

Chi-square tests of independence and additional plots were used to assess the strength of the relationship between illness group and the two secondary outcomes, loneliness and symptom burden.

All analyses were conducted using R, Version 3.6.3 (R Foundation for Statistical Computing).

\section{Results}

\section{Participant characteristics}

Patients recruited through April 3, 2020 were eligible for inclusion. We identified 13,872 patients with advanced illness criteria across $18 \mathrm{ED}$ sites nationwide who were assessed further for eligibility. Of those patients, 1138 (8\%) met all inclusion and no exclusion criteria and were eligible; $530(47 \%)$ of those eligible were enrolled, and 519 were randomized (Fig. 1).

Ten patients were found to meet exclusion criteria after enrollment and before randomization and were never randomized. Nineteen of those randomized were determined to be screen failures post-randomization, and were excluded from the analysis. Thus, the baseline analysis and tables comprise a total of 500 study patients.

\section{Participant demographics}

Baseline demographic characteristics are listed in Table 1. The cohort is $54 \%$ female, with a median age of 66 (IQR: 50 to 95 ) years, two-thirds white, $88 \%$ non-Hispanic, $89 \%$ United States-born, and 56\% have at least some college education. Approximately three-quarters practice a religious faith. Thirty-eight percent have an annual household income of less than $\$ 25,000$, and $32 \%$ are insured through Medicare only; $18 \%$ are dual eligible Medicare/Medicaid.

Approximately one-third are married, half are private home-dwellers, and about half (51\%) require assistance to care for themselves. Fifty percent have a primary family caregiver, which is defined as someone who provides regular assistance to the patient and is either a family member or a close friend who lives with the patient fulltime [31].

\section{Data Missingness}

For the demographic data, missing data was largely limited to annual household income, where $17 \%(n=83)$ of the patients either did not know or declined to report it. There were some other demographic items that patients declined to report, including level of education $(n=15)$, religious status $(n=11)$, ethnicity $(n=5)$, race $(n=4)$, marital status $(n=4)$, functional status $(n=2)$, and birth country $(\mathrm{n}=1)$.

There was virtually no missing data for the baseline survey data, where less than $1 \%(n=5)$ of patients did not provide sufficient data to calculate an overall FACT$\mathrm{G}$ score. Due to time constraints in the ED setting, two patients did not complete any questions in the UCLA 3item loneliness scale, three additional patients declined to answer the question 'I have a lack of companionship', one patient declined to answer 'I feel left out', and one declined to answer 'I feel isolated from others'. Also due to time constraints in the ED setting, one patient did not complete any questions in the ESAS-r.

\section{Baseline primary outcomes}

Out of a possible overall FACT-G score of 108, our cohort of persons presenting to the ED with end-stage illness have an overall median FACT-G score of 63 (IQR: 11 to 107) indicating compromised QOL. The subgroup of patients with advanced cancer have less compromised QOL across all four domains (physical, emotional, social/family, and functional) as well as a less compromised total score compared to patients with end-stage organ failure (Fig. 2). Those with end-stage organ failure have a median score of 59 (11 to 107), while those with cancer have a median score of 69 (12 to 106).

The linear regression model (Table 2) based on 475 patients with complete data suggests that illness type is indeed associated with QOL after adjusting for age, education level, race, sex, immigrant status, the presence of a caregiver, and hospital setting. On average, those with end-stage organ failure have lower QOL compared to those with advanced cancer, with an estimated difference of 9.6 points ( $95 \%$ CI: $5.9,13.3$ ), and those with at least two life-limiting illnesses have a further reduction of 7.4 points (95\% CI: 2.4, 12.5). This estimated difference between cancer and end-stage organ failure in overall score is consistent with the mean difference in Fig. 2. Older adults on average report better QOL than those of younger age after adjusting for all other characteristics; for each additional year of age, there is on average a 0.24 point increase (95\% CI: $0.07,0.41)$ in FACT-G score. The parameter estimates for the remaining 


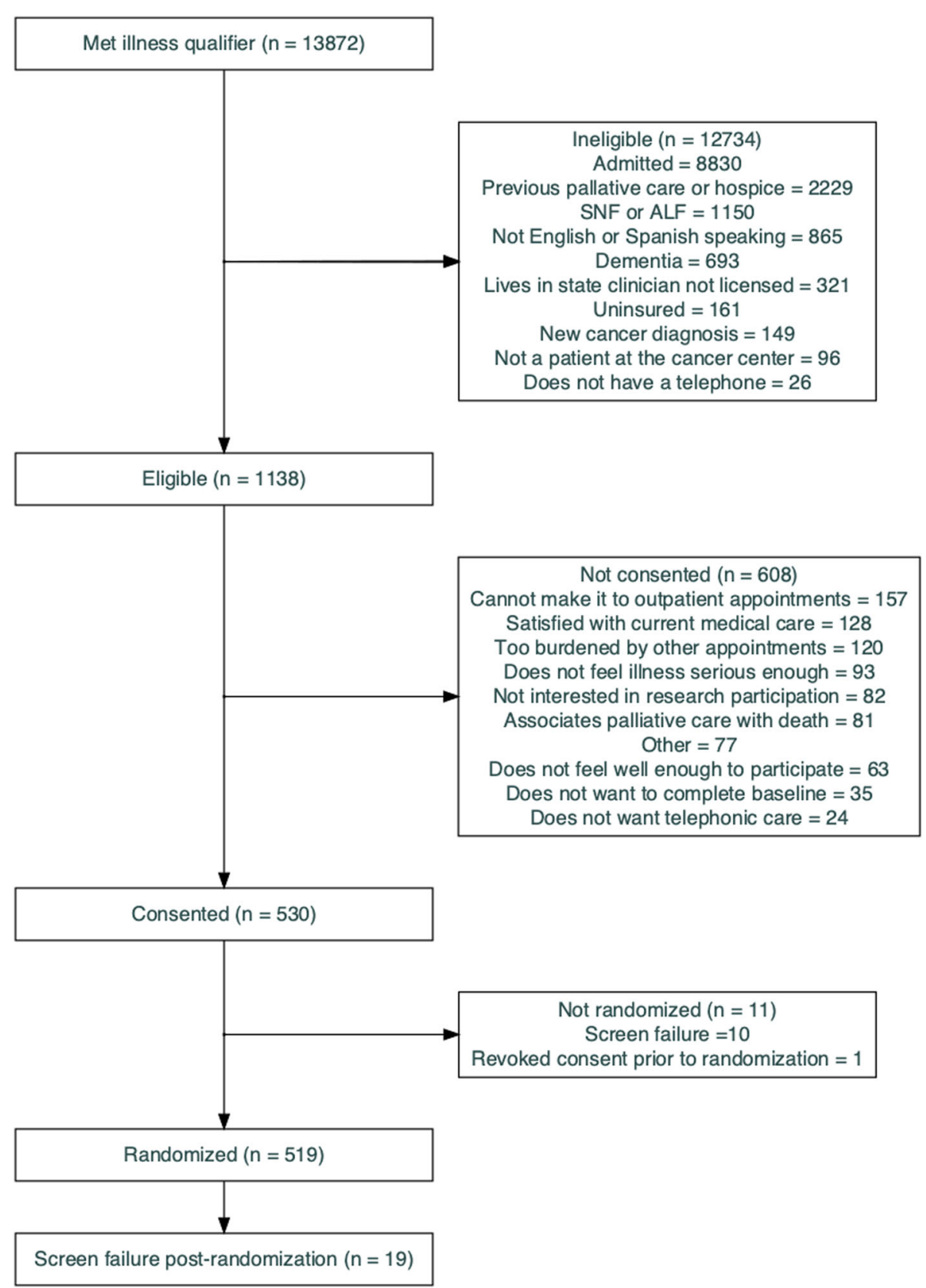

Fig. 1 Consolidated Standards of Reporting Trials (CONSORT) Diagram

characteristics were less conclusive. Overall, $8 \%$ of the variability in the outcome is accounted for by the set of covariates in our model.

\section{Baseline secondary outcomes}

Each of the three questions on the UCLA 3-item loneliness scale was examined separately (Fig. 3 - Panel A). Ten percent of cancer patients often felt isolated, versus $25 \%$ of end-stage organ failure patients. Similar patterns were seen for the other two loneliness items (9\% versus $26 \%$ often felt left out; $13 \%$ versus $25 \%$ lacked companionship). Patients with cancer appear to be more likely to have mild symptom burden (37\% vs $27 \%$ ) and less likely to have severe symptom burden compared with end-stage organ failure patients (9\% vs 17\%) (Fig. 3 - Panel B).

\section{Discussion}

This report demonstrates that the EMPallA randomized controlled trial is enrolling a diverse sample of older adults with advanced illness who present to the ED. In the EMPallA trial cohort, $59 \%$ of patients identified as 
Table 1 Baseline Characteristics of Participants

\begin{tabular}{|c|c|}
\hline Variable & $\begin{array}{l}\text { Totals }(N=500 \text {, } \\
\%)\end{array}$ \\
\hline Age (50-95) & 67 (10) (mean, SD) \\
\hline \multicolumn{2}{|l|}{ Sex } \\
\hline Female & $270(54)$ \\
\hline Male & $230(46)$ \\
\hline \multicolumn{2}{|l|}{ |llness* } \\
\hline Cancer & $206(41)$ \\
\hline End-stage renal disease & $101(20)$ \\
\hline Chronic obstructive pulmonary disease & $135(27)$ \\
\hline Congestive heart failure & $125(25)$ \\
\hline \multicolumn{2}{|l|}{ Comorbid (two or more of above Illnesses) } \\
\hline Yes & $63(13)$ \\
\hline No & $437(87)$ \\
\hline \multicolumn{2}{|l|}{ Race (4 Refused) } \\
\hline White & $294(59)$ \\
\hline Black & $147(29)$ \\
\hline Other & $44(9)$ \\
\hline Multiple races & $11(2)$ \\
\hline \multicolumn{2}{|l|}{ Ethnicity (5 Refused) } \\
\hline Hispanic & $54(11)$ \\
\hline Non-Hispanic & $441(88)$ \\
\hline \multicolumn{2}{|l|}{ Functional Status (2 Refused) } \\
\hline Disabled & $35(7)$ \\
\hline Requires considerable assistance & $83(17)$ \\
\hline Requires occasional assistance & $137(27)$ \\
\hline Cares for self, unable to do normal activity & $107(21)$ \\
\hline Normal activity & $136(27)$ \\
\hline \multicolumn{2}{|l|}{ Primary Language } \\
\hline English & $489(98)$ \\
\hline Spanish & $11(2)$ \\
\hline \multicolumn{2}{|l|}{ Income (30 Refused, 53 Don't Know) } \\
\hline Less than $\$ 25 \mathrm{~K}$ & $191(38)$ \\
\hline$\$ 25 K-\$ 49,999 K$ & $101(20)$ \\
\hline$\$ 50 K-\$ 99,999 \mathrm{~K}$ & $69(14)$ \\
\hline$\$ 100 \mathrm{~K}$ or more & $56(11)$ \\
\hline \multicolumn{2}{|l|}{ Education Level (1 missing, 15 Refused) } \\
\hline$<$ High school degree & $75(15)$ \\
\hline High school degree & $127(25)$ \\
\hline Some college/AA degree & $144(29)$ \\
\hline College degree or > & $138(28)$ \\
\hline \multicolumn{2}{|l|}{ Marital Status (4 Refused, 1 Other) } \\
\hline Married & $180(36)$ \\
\hline Never married & $111(22)$ \\
\hline Widow (er) & $83(17)$ \\
\hline
\end{tabular}

Table 1 Baseline Characteristics of Participants (Continued)

\begin{tabular}{|c|c|}
\hline Variable & $\begin{array}{l}\text { Totals }(N=500 \text {, } \\
\%)\end{array}$ \\
\hline Separated & $24(5)$ \\
\hline Divorced & $77(15)$ \\
\hline Living with a partner & $20(4)$ \\
\hline \multicolumn{2}{|l|}{ Residence type } \\
\hline Apartment, elevator & $125(25)$ \\
\hline Apartment, no elevator & $79(16)$ \\
\hline Private home with stairs & $194(39)$ \\
\hline Private home with no stairs & $81(16)$ \\
\hline Other & $21(4)$ \\
\hline \multicolumn{2}{|l|}{ Insurance Type (can have multiple) } \\
\hline Employer & $49(10)$ \\
\hline Purchased & $13(3)$ \\
\hline Medicare & $158(32)$ \\
\hline Medicaid & $65(13)$ \\
\hline Veterans Affairs & $3(1)$ \\
\hline Other & $18(4)$ \\
\hline Dual eligible Medicare/Medicaid & $89(18)$ \\
\hline $\begin{array}{l}\text { Dual eligible (any other than Medicare/ } \\
\text { Medicaid) }\end{array}$ & $105(21)$ \\
\hline \multicolumn{2}{|l|}{ Religion (11 Refused) } \\
\hline Do not practice/believe & $138(28)$ \\
\hline Catholic & $119(24)$ \\
\hline Other & $115(23)$ \\
\hline Protestant & $96(19)$ \\
\hline Jewish & $21(4)$ \\
\hline \multicolumn{2}{|l|}{ Born in US (1 Refused) } \\
\hline Yes & $447(89)$ \\
\hline No & $52(10)$ \\
\hline \multicolumn{2}{|l|}{ Has primary family caregiver } \\
\hline Yes & $250(50)$ \\
\hline No & $250(50)$ \\
\hline \multicolumn{2}{|l|}{ Hospital setting } \\
\hline Urban & $346(69)$ \\
\hline Suburban & $154(31)$ \\
\hline
\end{tabular}

*Patients can have multiple illnesses

white and $11 \%$ identified as Hispanic or Latino. Female participants made of $54 \%$ of the cohort. Thirty-two percent of study participants had Medicare only, and $18 \%$ were dual-eligible for Medicare and Medicaid. Participants were distributed between the diseases of interest; cancer was the most common diagnosis at $41 \%$ and ESRD the least common at 20\%. Notably, the EMPallA trial includes patients with multiple chronic conditions, whom are often excluded from randomized controlled trials [33]. Thirteen percent of study participants met 


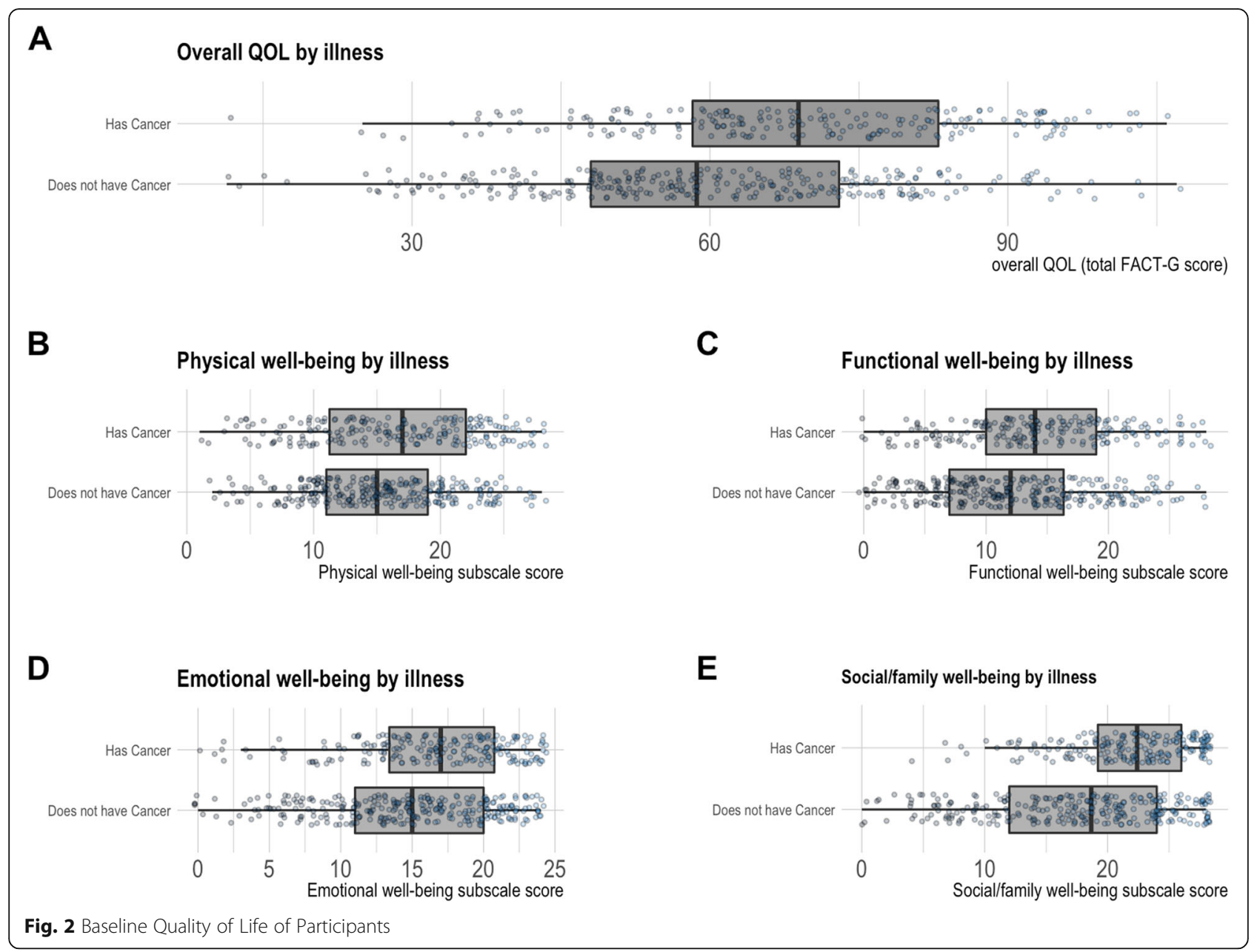

Table 2 Linear regression to examine the importance of illness group membership in predicting FACT-G score [32].

\begin{tabular}{ll}
\hline & $\begin{array}{l}\text { Dependent variable: } \\
\text { Total FACT-G score }\end{array}$ \\
\hline Age & $0.24(0.07,0.41)$ \\
Some college or more & $0.95(-2.45,4.35)$ \\
Has a caregiver & $1.28(-2.03,4.58)$ \\
Suburban hospital setting & $3.78(-0.002,7.57)$ \\
Non-white & $1.84(-1.96,5.64)$ \\
Male & $1.38(-1.91,4.67)$ \\
Foreign born & $1.32(-4.38,7.01)$ \\
Not comorbid & $7.42(2.40,12.45)$ \\
Has cancer & $9.62(5.90,13.34)$ \\
Constant & $49.71(43.43,55.98)$ \\
Observations & 475 \\
$R^{2}$ & 0.10 \\
Adjusted R & 0.08 \\
Residual Std. Error & $17.78(\mathrm{df}=465)$ \\
F Statistic & $5.58(\mathrm{df}=9 ; 465)$ \\
\hline
\end{tabular}

eligibility criteria for two or more serious, life-limiting illnesses, and we suspect that many more have multiple conditions that are less severe. The demographics of the patients in the EMPallA study are similar to those reported in 2017 by the Centers for Disease Control and Prevention from a representative sample of ED visits in the US [34].

In this large, multi-center randomized controlled trial in ED patients, we observed important differences in baseline QOL when stratifying by disease type and controlling for demographic variables. QOL was low for all study participants, which is consistent with their advanced illness status and suggests they may particularly benefit from palliative care interventions. When comparing illness types, patients with organ failure had worse QOL on all FACT-G subscales and the overall FACT-G score as compared with cancer patients. Additionally, patients with organ failure had higher symptom burden and more loneliness than cancer patients. One reason this might be the case is that cancer patients presenting to the ED have previously been healthy and are just starting the rapid end-of-life decline in functional status, 


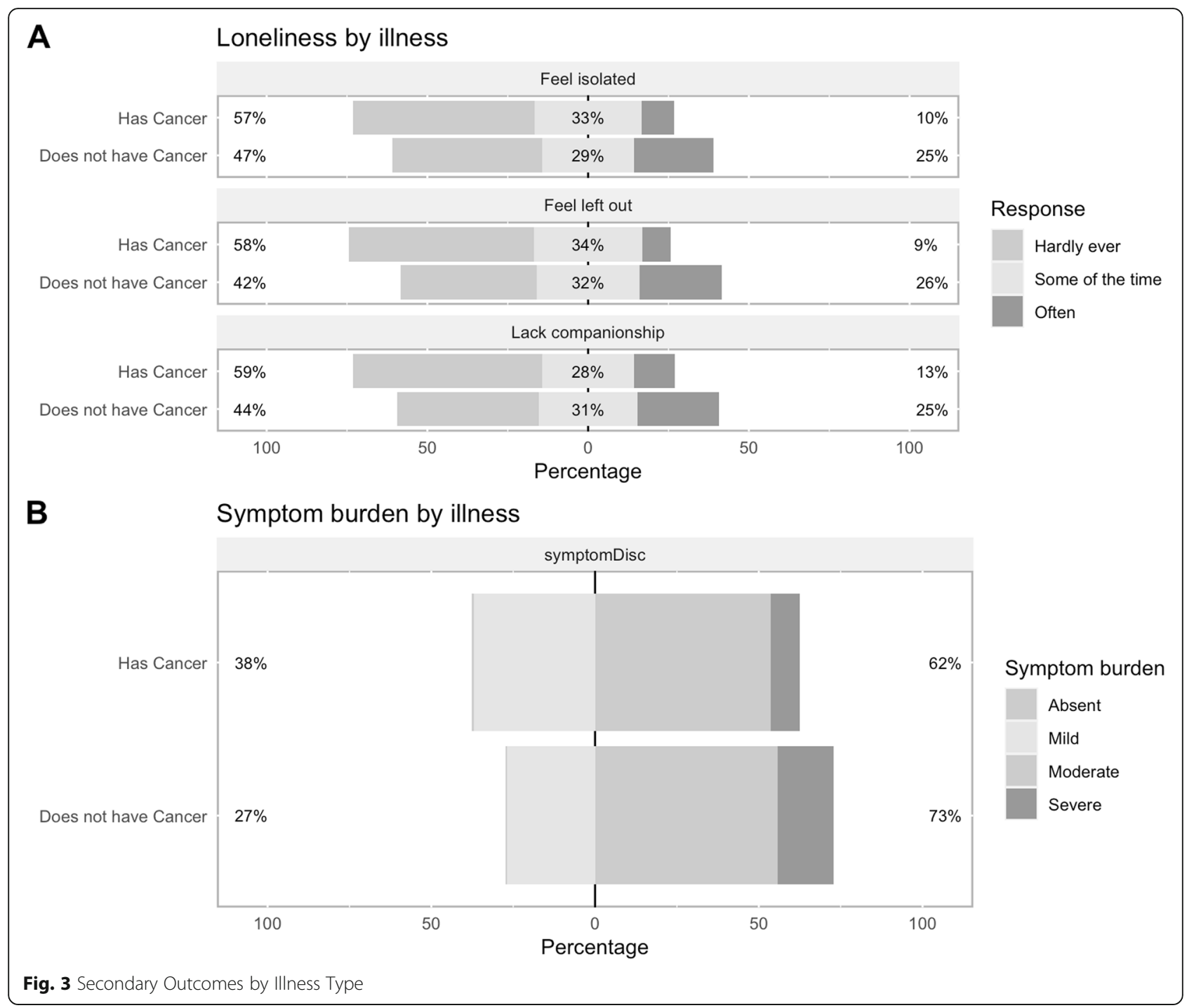

whereas organ failure patients have experienced repeated episodes of exacerbations and remissions [19]. Patients with $\mathrm{CHF}$ and COPD have been shown to have more hospital admissions and ED visits than cancer patients in the last months of life, which could contribute to poorer QOL [35]. Similarly, patients with ESRD tend to have lower rates of advance care planning, higher treatment intensity, and similarly high symptom burden in the last year of life compared to cancer patients [36].

Our model also indicated that meeting inclusion criteria for multiple advanced illnesses was independently associated with a poorer QOL. This is consistent with prior studies; a meta-analysis of 74 studies showed that an individual's QOL decreases with each additional illness [37]. Additionally, we found that older age is associated with improved QOL. We are unsure about why this is, but hypothesize it might be because patients who have lived longer have likely experienced less serious illness during their lifetime.

To our knowledge, this is the first large, multicenter trial enrolling older adults with multiple different advanced illnesses from the ED. While the distinct disease trajectories of patients with cancer and end-stage organ failure have been elucidated in other settings, this report is unique in presenting a snapshot of the distinct trajectories at the time of an ED visit. The differences by illness type in QOL, symptom burden, and loneliness seem to reflect the sudden deterioration of cancer patients at the end of life versus the undulating decline of those with organ failure [19].

For older adults with serious illness presenting to the ED, we have demonstrated that QOL, symptom burden, and loneliness differ by illness type, suggesting that palliative care needs may differ by disease 
category as well [38]. For this reason, the subgroup analyses by illness type in the final EMPallA analysis will be important in determining how to interpret the primary outcome of the study for patients of different illness types and in designing future palliative care interventions that benefit particular disease types. Despite similar symptom burdens, illness experience, and survival rates between advanced cancer and end-stage organ failure, much of the attention directed toward improving end-of-life care has focused on cancer patients $[17,35,36,39]$. This report highlights the need for more disease-specific research to improve patientcentered outcomes for those with end-stage organ failure presenting to the ED. Additionally, since patients with multiple chronic conditions have particularly low QOL, future research should focus on developing palliative care interventions to target these patients, as well as screening and referral protocols to be used by ED providers.

A strength of our study is that it is enrolling a large national cohort with a rich set of baseline demographic variables and patient-reported outcome measures. Its inclusive inclusion criteria facilitate recruitment of diverse patients. Additionally, by including patients with both cancer and end-stage organ failure, palliative care interventions can be assessed in unique disease processes.

Nevertheless, our study has various limitations. Only about $50 \%$ of patients who are approached are willing to enroll in the study. Patient refusal or failure to follow up could result in a non-response bias, where enrolled patients are those open to trying palliative care. Additionally, this study is limited to four specific diseases, only recruits English- and Spanish-speaking patients, and is conducted within the US, meaning it may not be generalizable to patients outside these demographics. Finally, all outcomes are self-reported rather than objectively measured. However, patient-reported outcomes are commonly used in palliative care and are validated to measure QOL and symptom burden [24, 25].

\section{Conclusions}

The EMPallA trial is enrolling a diverse sample of ED patients. The lower QOL, increased symptom burden, and increased loneliness found in end-stage organ failure patients versus cancer patients demonstrates how distinct disease trajectories manifest in the ED.

\section{Abbreviations}

ED: Emergency department; QOL: Quality of life; FACT-G: Functional Assessment of Cancer Therapy-General; EMPallA: Emergency Medicine Palliative Care Access; COPD: Chronic obstructive pulmonary disease; CHF: Congestive heart failure; ESRD: End-stage renal disease; IRB: Institutional review board; PCORI: Patient-Centered Outcomes Research Institute; RCT: Randomized controlled trial; US: United States; HER: Electronic health record; RA: Research assistant; ESAR-r: Edmonton Revised Symptom Assessment Scale

\section{Acknowledgements}

We would like to thank Nina Siman and the members of the EMPallA Study Advisory Committee for their contribution to the development of the manuscript.

The EMPallA Investigators study group contains the following authors: Caroline Blaum, MD.

Jason Bischof, MD.

Kei Ouchi, MD, MPH.

Marie-Carmelle Elie, MD, RDMS, FACEP, FCCM.

Robert Swor, DO.

Karen Jubanyik, MD.

Jeffrey T. Berger, MD.

Bharath Chakravarthy, MD, MPH.

Richelle J. Cooper, MD.

Christopher J. Coyne, MD.

Chinwe H. Ogedegbe, MD.

Isabel Castro, MPH.

Holden Caplan.

Simar Randhawa, MD, MPH.

Jordan Carpenter.

Nikita Umale, MBBS, MPH.

Rebecca Murray, CCRC, CBIS

Matthew Shaw.

Nora Daut, MPH.

Jennifer Bonito.

Nancy Hernandez.

Julia Vargas.

Alexandrea Cronin.

Diana McCarthy, RN, MSN.

\section{Authors' contributions}

All authors were involved in the study conception and design. AMS, MF, and JC collected study data. AMS, MF, KG, and CG analyzed and interpreted study data. AMS, MF, JC, and CG drafted the manuscript. AMS, MF, JC, KG, and CG critically revised the manuscript for important intellectual content. KG provided statistical expertise. CG obtained funding and supervised the study. JG and CG provided administrative, technical, and material support. All authors read and approved the final manuscript.

\section{Funding}

This work was funded through a Patient-Centered Outcomes Research Institute (PCORI) Award (PLC-1609-36306). The funders had a role in study design, data collection, and decision to publish.

\section{Availability of data and materials}

The data that support the findings of this study are available from PCORI but restrictions apply to the availability of these data, which were used under license for the current study, and so are not publicly available. Data are however available from the authors upon reasonable request and with permission of PCORI.

\section{Declarations}

Ethics approval and consent to participate

This study was approved by the New York University School of Medicine Institutional Review Board (study ID s17-01211). Informed consent was collected for all study participants at the time of enrollment. Before March 2020, consent was written only, but during the COVID-19 pandemic we obtained the ability to consent patients verbally, which was approved by the above Institutional Review Board.

\section{Consent for publication}

Not applicable.

\section{Competing interests}

The authors declare that they have no competing interests.

\section{Author details}

${ }^{1}$ Sidney Kimmel Medical College at Thomas Jefferson University, Philadelphia, PA, USA. ${ }^{2}$ Ronald O. Perelman Department of Emergency Medicine, NYU School of Medicine, New York University Langone Health, 227 E 30th Street, 
First Floor, New York, NY 10016, USA. ${ }^{3}$ Department of Population Health, New York University School of Medicine, 227 E 30th Street, First Floor, New York, NY 10016, USA.

Received: 2 July 2020 Accepted: 29 June 2021

Published online: 12 July 2021

\section{References}

1. Smith AK, McCarthy E, Weber E, Cenzer IS, Boscardin J, Fisher J, et al. Half of older Americans seen in emergency department in last month of life; Most admitted to hospital, And Many Die There. Health Affairs. 2012:31(6):1277_ 85. https://doi.org/10.1377/hlthaff.2011.0922.

2. Smith AK, Fisher J, Schonberg MA, Pallin DJ, Block SD, Forrow L, et al. Am doing the right thing? Provider perspectives on improving palliative care in the emergency department. Ann Emerg Med. 2009;54(1):86-93, e1. https:// doi.org/10.1016/j.annemergmed.2008.08.022.

3. Chan GK. End-of-life and palliative care in the emergency department: a call for research, education, policy and improved practice in this frontier area. J Emerg Nurs. 2006;32(1):101-3. https://doi.org/10.1016/j.jen.2005.10.006

4. National Consensus Project for Quality Palliative Care. Clinical practice guidelines for quality palliative care, executive summary. J Palliat Med. 2004; 7(5):611-27.

5. Elsayem A, Swint K, Fisch MJ, Palmer JL, Reddy S, Walker P, et al. Palliative care inpatient service in a comprehensive cancer center: clinical and financial outcomes. J Clin Oncol. 2004;22(10):2008-14. https://doi.org/10.12 00/JCO.2004.11.003.

6. Smith TJ, Coyne P, Cassel B, Penberthy L, Hopson A, Hager MA. A highvolume specialist palliative care unit and team May reduce in-hospital endof-life care costs. J Palliat Med. 2003;6(5):699-705. https://doi.org/10.1089/1 09662103322515202

7. Higginson IJ, Finlay I, Goodwin DM, Cook AM, Hood K, Edwards AGK, et al. Do Hospital-Based Palliative Teams Improve Care for Patients or Families at the End of Life? 2002;23(2):96-106.

8. Higginson IJ, Finlay IG, Goodwin DM, Hood K, Edwards AGK, Cook A, et al. Is there evidence that palliative care teams Alter end-of-life experiences of patients and their caregivers? J Pain Symptom Manag. 2003;25(2):150-68. https://doi.org/10.1016/S0885-3924(02)00599-7.

9. Manfredi PL, Morrison RS, Morris J, Goldhirsch SL, Carter JM, Meier DE. Palliative Care Consultations. 2000:20(3):166-73.

10. Casarett D, Johnson M, Smith D, Richardson D. The Optimal Delivery of Palliative Care. Archiv Internal Med. 2011;171(7):649-55.

11. Morrison RS. Cost savings associated with US Hospital palliative care consultation programs. Arch Intern Med. 2008;168(16):1783-90. https://doi. org/10.1001/archinte.168.16.1783.

12. Bakitas M, Lyons KD, Hegel MT, Balan S, Brokaw FC, Seville J, et al. Effects of a palliative care intervention on clinical outcomes in patients with advanced Cancer. JAMA. 2009;302(7):741-9. https://doi.org/10.1001/jama.2 009.1198 .

13. Wright AA. Associations between end-of-life discussions, patient mental health, medical care near death, and caregiver bereavement adjustment. JAMA. 2008;300(14):1665-73. https://doi.org/10.1001/jama.300.14.1665.

14. Wright AA, Keating NL, Balboni TA, Matulonis UA, Block SD, Prigerson HG. Place of death: correlations with quality of life of patients with cancer and predictors of bereaved caregivers' mental health. J Clin Oncol. 2010;28(29): 4457-64. https://doi.org/10.1200/JCO.2009.26.3863.

15. May P, Garrido MM, Cassel JB, Kelley AS, Meier DE, Normand C, et al. Prospective cohort study of hospital palliative care teams for inpatients with advanced Cancer: earlier consultation is associated with larger cost-saving effect. J Clin Oncol. 2015;33(25):2745-52. https://doi.org/10.1200/JCO.2014. 60.2334 .

16. Rabow MW, Dibble SL, Pantilat SZ, McPhee SJ. The comprehensive care team. Arch Intern Med. 2004;164(1):83-91. https://doi.org/10.1001/archinte.1 64.1.83.

17. Solano JP, Gomes B, Higginson IJ. A comparison of symptom prevalence in far advanced Cancer, AIDS, heart disease, chronic obstructive pulmonary disease and renal disease. J Pain Symptom Manag. 2006;31(1):58-69. https:// doi.org/10.1016/j.jpainsymman.2005.06.007.

18. Hofstede JM, Raijmakers NJ, Van Der Hoek LS, Francke AL. Differences in palliative care quality between patients with cancer, patients with organ failure and frail patients: a study based on measurements with the consumer quality index palliative care for bereaved relatives. Palliat Med 2016:30(8):780-8. https://doi.org/10.1177/0269216315627123.

19. Lunney JR. Patterns of functional decline at the end of life. JAMA. 2003; 289(18):2387-92. https://doi.org/10.1001/jama.289.18.2387.

20. George N, Phillips E, Zaurova M, Song C, Lamba S, Grudzen C. Palliative Care Screening and Assessment in the Emergency Department: A Systematic Review. J Pain Symptom Manage. 2016;51(1):108-19.e2.

21. Grudzen CR, Shim DJ, Schmucker AM, Cho J, Goldfeld KS. Emergency medicine palliative care access (EMPallA): protocol for a multicentre randomised controlled trial comparing the effectiveness of specialty outpatient versus nurse-led telephonic palliative care of older adults with advanced illness. BMJ Open. 2019;9(1):e025692. https://doi.org/10.1136/ bmjopen-2018-025692.

22. Harris PA, Taylor R, Minor BL, Elliott V, Fernandez M, O'Neal L, et al. The REDCap consortium: Building an international community of software platform partners. J Biomed Inform. 2019;95:103208.

23. Harris PA, Taylor R, Thielke R, Payne J, Gonzalez N, Conde JG. Research electronic data capture (REDCap)--a metadata-driven methodology and workflow process for providing translational research informatics support. J Biomed Inform. 2009;42(2):377-81. https://doi.org/10.1016/j.jbi.2008.08.010.

24. Cella DF, Tulsky DS, Gray G, Sarafian B, Linn E, Bonomi A, et al. The functional assessment of Cancer therapy scale: development and validation of the general measure. J Clin Oncol. 1993;11(3):570-9. https://doi.org/10.12 00/JC0.1993.11.3.570.

25. McCaffrey N, Bradley S, Ratcliffe J, Currow DC. What Aspects of Quality of Life Are Important From Palliative Care Patients' Perspectives? A Systematic Review of Qualitative Research. J Pain Symptom Manag. 2016;52(2):318-28.e5.

26. Hughes ME, Waite LJ, Hawkley LC, Cacioppo JT. A short scale for measuring loneliness in large surveys: results from two population-based studies. Res Aging. 2004;26(6):655-72. https://doi.org/10.1177/0164027504268574.

27. Russell D, Peplau LA, Ferguson ML. Developing a measure of loneliness. J Pers Assess. 1978;42(3):290-4. https://doi.org/10.1207/s15327752jpa4203_11.

28. Chang VT, Hwang SS, Feuerman M. Validation of the Edmonton symptom assessment scale. Cancer. 2000;88(9):2164-71. https://doi.org/10.1002/(SICI)1 097-0142(20000501)88:9<2164::AID-CNCR24>3.0.CO;2-5.

29. Zimmermann C, Burman D, Bandukwala S, Seccareccia D, Kaya E, Bryson J, et al. Nurse and physician inter-rater agreement of three performance status measures in palliative care outpatients. Support Care Cancer. 2010;18(5): 609-16. https://doi.org/10.1007/s00520-009-0700-9.

30. Hui D, Kilgore K, Fellman B, Urbauer D, Hall S, Fajardo J, et al. Development and cross-validation of the in-hospital mortality prediction in advanced cancer patients score: a preliminary study. J Palliat Med. 2012;15(8):902-9. https://doi.org/10.1089/jpm.2011.0437.

31. Application for Comprehensive Assistance for Family Caregivers Program2020. Available from: https://www.va.gov/vaforms/medical/pdf/10-10CG.pdf.

32. Hlavac M. Stargazer: Well-Formatted Regression and Summary Statistics Tables 2018. Available from: https://CRAN.R-project.org/package=stargazer.

33. Buffel Du Vaure C, Dechartres A, Battin C, Ravaud P, Boutron I. Exclusion of patients with concomitant chronic conditions in ongoing randomised controlled trials targeting 10 common chronic conditions and registered at ClinicalTrials.gov: a systematic review of registration details. BMJ Open. 2016; 6(9):e012265.

34. Rui P, Kang K. National Hospital Ambulatory Medical Care Survey: 2017 emergency department summary tables. National Center for Health Statistics; 2017.

35. Lastrucci V, D'Arienzo S, Collini F, Lorini C, Zuppiroli A, Forni S, et al. Diagnosis-related differences in the quality of end-of-life care: a comparison between cancer and non-cancer patients. PLoS One. 2018;13(9):e0204458. https://doi.org/10.1371/journal.pone.0204458.

36. Wachterman MW, Lipsitz SR, Lorenz KA, Marcantonio ER, Li Z, Keating NL. End-of-life experience of older adults dying of end-stage renal disease: a comparison with Cancer. J Pain Symptom Manag. 2017;54(6):789-97. https://doi.org/10.1016/j.jpainsymman.2017.08.013.

37. Makovski TT, Schmitz S, Zeegers MP, Stranges S, Van Den Akker M. Multimorbidity and quality of life: systematic literature review and metaanalysis. Ageing Res Rev. 2019;53:100903. https://doi.org/10.1016/j.arr.2019. 04.005 .

38. Huijberts S, Buurman BM, de Rooij SE. End-of-life care during and after an acute hospitalization in older patients with cancer, end-stage organ failure, or frailty: a sub-analysis of a prospective cohort study. Palliat Med. 2016; 30(1):75-82. https://doi.org/10.1177/0269216315606010. 
39. Penders YW, Onwuteaka-Philipsen B, Moreels S, Donker GA, Miccinesi G, Alonso TV, et al. Differences in primary palliative care between people with organ failure and people with cancer: an international mortality follow-back study using quality indicators. Palliat Med. 2018;32(9):1498-508. https://doi. org/10.1177/0269216318790386.

\section{Publisher's Note}

Springer Nature remains neutral with regard to jurisdictional claims in published maps and institutional affiliations.

Ready to submit your research? Choose BMC and benefit from:

- fast, convenient online submission

- thorough peer review by experienced researchers in your field

- rapid publication on acceptance

- support for research data, including large and complex data types

- gold Open Access which fosters wider collaboration and increased citations

- maximum visibility for your research: over $100 \mathrm{M}$ website views per year

At $\mathrm{BMC}$, research is always in progress.

Learn more biomedcentral.com/submissions 